\title{
Make Potsdam great again
}

André Tomczak

Manuel Lutz

Holger Zschoge
2018: Potsdam im Umbruch - der Mitte-Komplex

Eindrucksvolle Rekonstruktionsprojekte, ein Kahlschlag im Bestand der Nachkriegsmoderne und ein eskalierter Wohnungsmarkt: die Entwicklung der kleinen Groß-, Landeshaupt- und Welterbestadt sorgt kontinuierlich für Schlagzeilen. Den Titel dieses Beitrags inspirierend, schrieb die Frankfurter Allgemeine Sonntagszeitung im April 2017 „Make Potsdam Schön Again“ (Maak/Seidel 2017), um die Auslöschung der urbane Mischung schaffenden DDR-Bauten zugunsten von fake-Fassaden anzuprangern. Ungetrübt von solcher Kritik wird der kosmetische Umbau der Potsdamer Stadtmitte mit dem zentralen Argument vorangetrieben, der durch und nach dem Krieg geschundene Stadtkörper müsse umfassend Heilung erfahren. Konkret bedeutet dies den Abriss unliebsamer Nachkriegsmoderne und eine neue Inszenierung als preußische Residenz. Mit rekonstruierten Schlossfassaden für den neuen Brandenburgischen Landtag (2014), mit dem privaten Kunstmuseum Barberini am Alten Markt (2016) und der Baustelle Garnisonkirche wird Potsdam zum Extrembeispiel: ein ganzes Stadtzentrum wird als nationales Denkmal rekonstruiert.

Diese prestigeorientierte Stadtentwicklung, vielmals ausgebremstes Engagement der Zivilgesellschaft und astronomische Mieten sorgen lokal dauerhaft für Konflikte und Kontroversen (vgl. Arte 2017). Doch die langjährigen Debatten über die Zukunft der Stadtmitte wurden im Sommer 2018 mit dem Abriss des unschätzbar potenzialreichen, 18.00o $\mathrm{m}^{2}$ großen Hochschulgebäudes der Fachhochschule (FH) am Alten Markt (ehemaliges Institut für Lehrerbildung, 1974) vorerst beendet. Mit dem Verlust des benachbarten Brauhausbergs - hier wurde der alte Landtag verkauft, mit einem Spaßbad die Ansicht verkorkst und die 1971 errichtete Schwimmhalle geschleift (Kil 2017) - drängen rekonstruierende Nachverdichtung und Verwertung bis ins landschaftlich geprägte Zentrum der Stadt. Die städtische Agenda, zentrale Gebäude der DDR abzureißen, bleibt ungebrochen: auf dieser Liste stehen das ehemalige Restaurant Minsk (1977), das Hotel Mercure 
(ehemaliges Interhotel Potsdam, 1969) und das seit 2015 als Kunst- und Kreativhaus zwischengenutzte Rechenzentrum (1971).

\section{Stadt von oben. Die Akteur_innen der Rekonstruktion}

Potsdams Mitte demonstriert Stadtentwicklung von oben par excellence und zeigt ausgeprägte Züge einer Re-Feudalisierung von Stadt. 29 Jahre nach der politischen Wende hat die zahlenmäßig überschaubare Bürgerinitiative „Mitteschön“ ihre Vision von Stadt - und damit ihre Version der Geschichte und Bedeutung - ungeachtet langjährigen Protests und entgegen aller Widersprüche durchgesetzt. Im Folgenden werden wir die Durchsetzung der Rekonstruktionsagenda in Potsdams Mitte skizzieren sowie zentrale Akteure und Entscheidungsprozesse mit ihren Hintergründen und Kontexten beleuchten, um auszuloten, wie diese Agenda zustimmungsfähig gemacht wurde und welche Effekte lokale Proteste und Alternativen hatten.

Impulsgeber und Wegbereiter dieser einseitig auf historische Qualitäten orientierten Stadtentwicklung sind einflussreiche Privatiers: SAP-Mitgründer und Multimilliardär Hasso Plattner veredelte den ohnehin geplanten Landtagsneubau in letzter Minute mit 20 Millionen Euro zur Schlossrekonstruktion, ermöglichte dessen Deckung in Kupfer und komplettierte die Rekonstruktion des Platzes durch sein Privatmuseum Palais Barberini. 15 Jahre zuvor hatte Günther Jauch mit seinem Baugeschenk des Fortunaportals die Idee, das Stadtschloss zu rekonstruieren, prominent und wegweisend in den damals noch recht leeren Stadtraum gestellt.[1] 2016 spendete Jauch erneut für den Abriss von Gebäuden, die er wie im Fall der FH als „,sozialistische Notdurftarchitektur" bezeichnet (rbb 2017). Mit seinen 1,5 Millionen Euro für das Projekt Garnisonkirche will er noch potentere Prominenz zu Großspenden bewegen.

\section{Stadt von unten. Recht auf Stadt oder Recht auf Debatte?}

Seit 2014 haben hunderte Kunst-, Kultur- und Kreativschaffende mit der Initiative „Kulturlobby“ ihren Anspruch auf Stadt deutlich gemacht und gegen den drohenden Verlust der letzten Kreativräume protestiert. Ein Jahr

\section{HAUS DER GEGENWART}

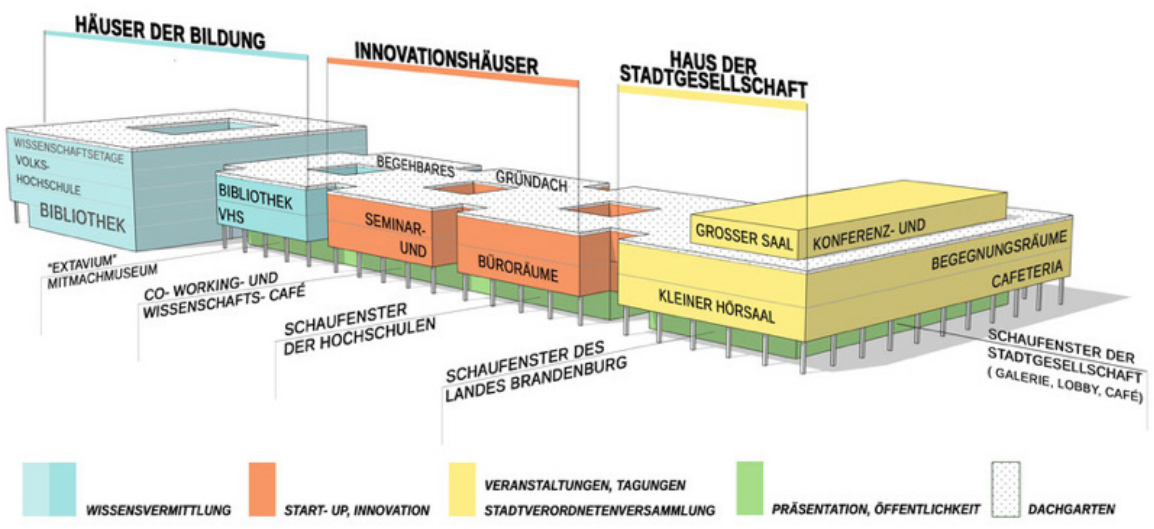

Abb. 1 Umnutzungskonzept für die $\mathrm{FH}$ (Quelle: Potsdamer Mitte neu denken 2016) 
später hinterfragte die Initiative „Potsdamer Mitte neu denken“ erstmals die Sinnhaftigkeit der Planungen für das 1999 eingeleitete Sanierungsgebiet Potsdamer Mitte. Damit fordern sie eine bis dato ausgebliebene Debatte über die Nachnutzungspotenziale des baulichen Erbes der DDR in Potsdams Innenstadt ein. Ein Nachnutzungskonzept verdeutlichte die enormen Chancen des FHGebäudes für die Stadtgesellschaft und den Innovationsstandort (vgl. Abb. 1).

Bemerkenswert ist, dass die Initiative zwar in erster Linie eine Debatte über mögliche Nutzungen und Funktionen, neu entstandene Bedarfe und offensichtliche Potenziale in der Stadtmitte anstoßen wollte, jedoch häufig vordergründig als ostmodern-nostalgische Initiative dargestellt wurde. Die für die Initiative tragenden Aspekte - Mitbestimmung in der Stadtentwicklung, Bewusstsein für kommunales Eigentum, Nachhaltigkeit blieben in der anhaltenden öffentlichen Debatte oft von Fragen von Ästhetik und Authentizität überlagert.

\section{Hintergründe: Die Potsdamer Mitte und ihr, verlorenes Herz}

Zum Mauerfall 1989 stand Potsdam vor großen städtebaulichen Herausforderungen: Während die Altbaubereiche durch Leerstand und Verfall geprägt waren, präsentierte sich das historische Zentrum unvollendet und großmaßstäblich. Die Bemühungen zur Ausbildung eines neuen, sozialistischen Zentrums hatten dort trotz zahlreicher ,Zentrumswettbewerbe' nur zu einem fragmentierten Ergebnis geführt: der von Protesten begleitete Abriss des schwer zerstörten Schlosses (1959/60) hatte eine zentrale Leerstelle hinterlassen, ein großräumiger Verkehrsknoten entstand. Dieser Situation begegneten die Stadtverordneten im Oktober 1990 mit einem Beschluss, der bis heute Grundsatzcharakter behalten hat:

„Der Magistrat wird beauftragt,

(1) energische Anstrengungen zu unternehmen, dem weiteren Verfall der verbliebenen echten historischen Bausubstanz Einhalt zu gebieten (2) eine langfristige, die Jahrtausendwende überschreitende Konzeption für eine von Verantwortung getragene behutsame Wiederannäherung an das charakteristische historisch gewachsene Stadtbild zu entwickeln.“ (Landeshauptstadt Potsdam 1990)

Während der Sanierungsstau in den Altbauvierteln unmittelbar angegangen wurde, sah man für den Bereich der alten Stadtmitte zunächst nur eine Art Stadtreparatur vor, die auf eine Neubesetzung der Schlossfläche und eine Wiederherstellung der mit ihr verbundenen stadträumlichen Bezüge fokussierte. Eine mögliche Rekonstruktion des Stadtschlosses wurde erst um die Jahrtausendwende Gegenstand städtischer Debatten. Nach einer Prüfung vergleichsweise banaler Nutzungen (Hotel, Wohnen, Museum) beschlossen Stadt und Land schließlich 2005, die Fläche als Sitz des Landtages zu nutzen.

Mit der Aussicht auf eine Neubebauung der Schlossparzelle wurden die Planungen für die Potsdamer Mitte 2005/o6 in der umstrittenen „Planungswerkstatt Potsdamer Mitte“ konkretisiert: sahen die ersten Konzepte noch eine behutsam ergänzende Bebauung mit Bezugnahme auf den historischen Stadtgrundriss vor, wurde nun festgelegt, dass das Fachhochschulgebäude und das benachbarte, Staudenhof genannte Wohngebäude komplett 
verschwinden und für eine annähernde Wiederherstellung der vormodernen Bebauungsstruktur Platz machen sollen. Hierfür hatte die Verwaltung ihre Wunschvorstellungen kurzerhand eigenmächtig in einen sogenannten Konsensplan zusammengelegt. Damit verloren die Planungen seit 2006 jeglichen Bezug zur zunächst beschworenen Behutsamkeit: die Wiederannäherung wurde radikal in eine weitestgehende Wiederherstellung der vormodernen, barocken Stadtstruktur uminterpretiert.

\section{Vertiefung: Garnisonkirche und Rechenzentrum}

Als Residenz- und Militärstadt war Potsdam direkt den preußischen Königen unterstellt - ist also immer Stadt von oben gewesen. Erst mit dem Kaiserreich entwickelte sich eine kommunale Selbstverwaltung und mit ihr eine Art Stadtbürgertum. Dennoch blieb Potsdam das Resultat fürstlicher Repräsentation - und entwickelte sich in der Weimarer Republik nicht nur zum Sehnsuchtsort der guten alten Zeit, sondern auch zum Symbolort national-konservativer und revanchistischer Gesinnung.

Besondere Brisanz erlangt diese Kontinuität angesichts der 2017 trotz anhaltender Proteste begonnenen Rekonstruktion der Garnisonkirche: Die frühere Staats- und Militärkirche Preußens, vor allem durch die Vereidigung Hitlers am 21. März 1933, dem ,Tag von Potsdam', und als Grablege Friedrich des Großen bekannt, stand bereits in der Kaiserzeit für die enge Verbindung zwischen Staat, Militär und Kirche. In der Zeit der Weimarer Republik avancierte sie so zum Symbolbau der extremen Rechten. Die Kirche wurde im Luftangriff 1945 schwer zerstört. Ihre Ruine verblieb bis zu ihrer Sprengung 1968 als mahnende und stadtbildprägende Dominante. Am gleichen Ort wurde wenig später das Rechenzentrum genannte, fünfgeschossige Datenverarbeitungszentrum mit dem denkmalgeschützten Sockelmosaik „Der Mensch bezwingt den Kosmos“ von Fritz Eisel errichtet.

Bemühungen zum Wiederaufbau der Garnisonkirche wurden kurioserweise 1983 in Westdeutschland aufgenommen: Fallschirmspringerleutnant Max Klaar rief in Isarlohn eine Traditionsgemeinschaft „Potsdamer Glockenspiel“ ins Leben, welche eben dieses nachgießen und 1990 nach Potsdam bringen ließ. Erst 2004 distanzierten sich kirchliche und gesellschaftliche Kreise vom rechtsextremen Militär Klaar und seinen Spendenmillionen: eine eigene Stiftung Garnisonkirche mit prominenten Mitgliedern aus Politik und Kirche bemüht sich seitdem um Mittel für die Rekonstruktion des Turms, welche im Oktober 2017 mit Jahren Verzug, ohne Gesamtfinanzierung und von Protesten begleitet, begonnen hat. Das benachbarte Rechenzentrum wird seit 2015 von über 250 Mietparteien als Kunst- und Kreativhaus zwischengenutzt - ein toller Erfolg der Initiative „Kulturlobby“ und bereits Leuchtturmprojekt (Pietryga 2018). Da aber Teile des Hauses auf dem Grundstück der Stiftung Garnisonkirche liegen, hängt die Weiternutzung von deren Zustimmung ab.

\section{Stadt von oben: Privatisierung und Verwertung}

Die zurückliegenden Auseinandersetzungen für Neudenken, also Alternativen zu Abriss, Privatisierung und Fassadenkultur, knüpfen an die elementaren Fragen heutiger Stadtentwicklung an: Wem gehört die Stadt - Wer 
kann mitentscheiden und mitgestalten? Seit dem Mauerfall hat sich in Potsdam eine lokale Bauallianz zwischen den Parteien der Rathauskoalition, der Stadtverwaltung, Projektentwickler_innen, Einzelinvestor_innen und ausführenden Firmen etabliert, die den Privatisierungskurs maßgeblich steuert. Während die kommunalen Wohnungsbestände kontinuierlich schrumpfen (20 Prozent in 2016, vgl. Landeshauptstadt Potsdam 2017a: 14), bewirbt sich die Stadt auf internationalen Real Estate Messen als attraktiver Investitionsstandort, an dem prominente Immobilieninvestor_innen investieren. So erreichen die Miet- und Wohnungskaufpreise jährlich neue Rekordhöhen: längst werden in Potsdam die mit Abstand teuersten Mieten Ostdeutschlands aufgerufen (laut Mietspiegel 2018 durchschnittlich 10,47 Euro $/ \mathrm{m}^{2}$ ). Mit durchschnittlichen Kaufpreisen von etwa 5.00o Euro $/ \mathrm{m}^{2}$ zählt Potsdam zu den zehn teuersten Städten Deutschlands (Zschiek 2018, Gellner 2018). Der Wohnungsleerstand liegt bei unter 1 Prozent.

Die Rekonstruktion der Stadtmitte folgt dem Prinzip dieser Privatisierungspolitik und setzt fort, was in den zentrumsnahen gründerzeitlichen Vierteln bereits weitgehend vollzogen wurde: die Lobby der privaten Eigentümer_innen und Entwickler_innen drängen auf Privatisierung, mit der sie hohe Gewinne erzielen und sehen sich unterstützt durch die Kommune, die mit dem Verkauf der öffentlichen Liegenschaften den Haushalt stabilisieren will. Das Ausmaß der Verflechtung von Immobilienwirtschaft und Stadtpolitik kann an verschiedenen Affären der letzten Jahre abgelesen werden, in denen die Medien publik machen, dass kommunale Wohnbestände oder Bauland unter dubiosen Umständen weit unter Wert an lokale Investoren wie Groth, Semmelhaack, Günter Jauch oder Wolfhard Kirsch verkauft werden (vgl. Tillack 2011, Hallo Potsdam 2017).

Doch während die steigenden Gewinnerwartungen und das routinierte Zusammenspiel dieser Wiederaufbau-Allianz die Entwicklung der Stadt bestimmen, sind die Spenden der privaten Akteure auch ein Indiz dafür, dass die Rekonstruktion der preußischen Stadtgestalt nicht auf ökonomische Interessen reduziert werden kann. Vielmehr soll an prominenter Stelle die Rechtfertigung der bisherigen Stadtentwicklungspolitik demonstriert werden: Der Verkauf städtischen Bodens gilt als unabdingbare Voraussetzung für gelungenen, weil schönen Stadtumbau. Mit der 2005 entstandenen Gruppierung „Bürger für die Mitte - Mitteschön!“ erhielt die privatisierende Rekonstruktion eine bürgerschaftlich-elitäre Legitimation (Förderverein Wiedergewinnung der Potsdamer Mitte e.V. 2018): Investor_innen gelten hier als engagierte und aufgeklärte Bürger_innen, die „ihren Lebensraum aktiv mitbestimmen wollen" und werden für ihr besonderes Engagement zum Wohle der Stadt gewürdigt (ebenda).

\section{Hintergrund: Stadt im Umbruch}

Zur Vorgeschichte der aktuellen Auseinandersetzungen für ein Recht auf Stadt gehören neben den Instandbesetzungen der Wendezeit auch anhaltende Jugendkulturprotesten um das Jahr 2008. Auslöser war die Schließung mehrerer Jugend- und soziokultureller Orte in der Innenstadt. Ein breiter Zusammenschluss protestierte gegen die Verdrängung junger Menschen und meldete vehement Anspruch auf die Stadtmitte an (Oelschläger 2014). Das 
2009 als Kompromiss ausgewiesene neue Jugendkulturzentrum ,freiLand“ zeigt ein typisches Muster der Befriedung: lautstarke Forderungen nach Räumen für Nichtkommerzielles, Kultur und Miteinander können politische Anerkennung erringen - solange sie nicht das Bild der schönen Stadt brechen und Standorte am Rand akzeptieren (freiLand 2018).

Zeitgleich entwickelten sich Überteuerung und schleichende Verdrängung zum dominanten Politikum. Seit 2008 haben sich mal Hunderte, mal Tausende zu „Mietenstopp-Demos“ zusammengefunden (AK Recht auf Stadt Potsdam 2013, Kramer 2017). Doch obwohl sich die Schar der Protestierenden beständig erweitert - zunehmend demonstrieren auch Studierende (Calvez 2018) und Kulturschaffende - entwickeln die Proteste nur langsam eine politische Dynamik. Potsdam, so ein aktuelles Studienergebnis des Wissenschaftszentrums Berlin für Sozialforschung (WZB), ist eine der am stärksten segregierten Städte Deutschlands (Helbig/Jähnen 2018). Die Kluft zwischen den gerade bei Zuzügler_innen beliebten zentrumsnahen Altbauvierteln und den DDR-Wohnsiedlungen ist unübersehbar. Zwar sind die Bewohner_innen der meist peripheren Plattenbaugebiete nur bedingt von steigenden Mieten bedroht, sehen sich von der Entwicklung der Stadt(mitte) jedoch zunehmend abgehängt. Der Vergleich des tatsächlich pulsierenden und des beschworenen verlorenen Zentrums markiert dies deutlich: Während entlang der Brandenburger Straße seit langem rege Betriebsamkeit herrscht, bleibt der neue Alte Markt verwaist.

Die Distanz der potenziell von Potsdams Restrukturierung betroffenen Stadtnutzer_innen zur politischen Debatte wird durch den Umstand verstärkt, dass Potsdams Bevölkerung seit 1990 zu zwei Dritteln ausgetauscht wurde und Potsdam bis heute eine ausgeprägte Pendlerstadt ist. Über 90 Prozent der Potsdamer Studierenden wohnen in Berlin. Zwar ist diese Gruppe von der Wohnungsnot direkt betroffen, da in den letzten Jahren ausschließlich hochpreisige Mikroapartments für Studierende gebaut wurden, doch mündet dies selten in Kritik an dem Narrativ ,Potsdam ist doch so schön‘. Der Anteil der Stadtbevölkerung, der (noch) Sehnsüchte nach einer Stadtmitte hegt, die mehr als Konsum bietet, schwindet stetig und wird politisch marginalisiert.

Das Ziel, die Stadt ,wieder schön zu machen' konnte so lange Zeit ohne große Kritik verfolgt werden. Mit ihm wurde und wird der Verkauf von Flächen und Immobilien an private Investoren gerechtfertigt, da angeblich nur sie die alte Pracht wiederherstellen können. Damit überschneiden sich hier klassische kommunale Wirtschaftsförderungsziele wie Tourismus und die ästhetischen Präferenzen wie auch Profitinteressen der Eliten nahtlos. Steigende Tourismuszahlen wie sie zuletzt in Folge der Eröffnung des wiederaufgebauten Barberini Palais vermeldet werden, werden von der Stadt als Bestätigung für den Privatisierungskurs interpretiert, die vergessen lassen, wie viel öffentliche Investition in der Rekonstruktion steckt, die für andere, gemeinwohlorientierte Stadtpolitik fehlt. Eklatantes Beispiel für verschleierte Subventionen privater Investoren ist die Garnisonkirche, deren Bau vollständig aus Spendengeldern finanziert werden sollte, bislang aber maßgeblich von der Stadt, dem Bund und der evangelischen Kirche getragen wird. 


\section{Stadt von unten: Wem gehört die Stadt?}

Wie beschrieben konnte erst 2015 mit den Aktivitäten der Initiative „Potsdamer Mitte neu denken“, mit dem Bürgerbegehren „Kein Ausverkauf der Potsdamer Mitte“ 2016 und dem 2017 gegründeten Bündnis „Stadtmitte für Alle“ eine lokale Debatte initiiert worden, die neben ästhetischen, städtebaulichen und erinnerungspolitischen Fragen auch die Frage nach den notwendigen und wünschenswerten Funktionen der Innenstadt stellt. Das 2018 abgerissene FH-Gebäude war die letzte große städtische Immobilie im Zentrum der Stadt, auf der die Stadt gemeinwohlorientierte Nutzungen ermöglichen konnte. Statt weiter bestehende Potentiale wie zum Beispiel auch das benachbarte zu DDR-Zeiten erbaute Wohnhaus Staudenhof mit über 180 Kleinwohnungen abzureißen, fordern die Initiativen Erhalt und Um-Nutzung.

Mit zahlreichen Veranstaltungen und kreativen Protestformen wie „Platz nehmen“ auf dem Alten Markt und einem „24-Stunden-Staffellauf“ um die Fachhochschule haben diese Initiativen ihre Forderung direkt in die Stadt getragen (vgl. Abb. 2). Diese Aktionen entfalteten eine starke diskursive und physische Mobilisierungskraft: im Laufe weniger Monate haben mehrere Tausend Menschen - oft zum ersten Mal - den Alten Markt als signifikanten Ort im Zentrum wahrgenommen und ihn sich angeeignet. Mit dem Bespielen des Platzes durch regelmäßige Versammlungen, dem Aufstellen von Sofas und Sitzkissen, Infoständen, Siebdruckwerkstätten, Spielen und Konzerten und einem Protestcamp wurde diese Leerstelle mit Leben gefüllt. Aus der Aneignungspraxis vieler oft bislang nicht politisierter Menschen entwickelten sich gemeinsame Visionen einer alternativen Stadtmitte. Vor allem die Fachhochschule wurde zu einer Projektionsfläche für Nutzungswünsche all jener, die sich mit der Verwertung und ,Preußifizierung' der Stadt unwohl fühlen. Die Initiative „Potsdamer Mitte Neu Denken“ hat in zahlreichen Diskussionen und Veranstaltungen nicht nur die denkmal- und städtebauliche Signifikanz der Fachhochschule in den lokalen Diskurs eingebracht sondern auch das Umnutzungspotential dieses Gebäudes betont. Die Sanierung, so die Forderung an die Stadt, ist möglich und kostengünstiger als der Abriss und ermöglicht die Errichtung eines „Haus der Stadtgesellschaft“, das

Abb. 2 Aktion „Platz nehmen" des Bündnis Stadtmitte für alle am 12.03.2018 (Quelle: Stadtmittefueralle.de 2018)

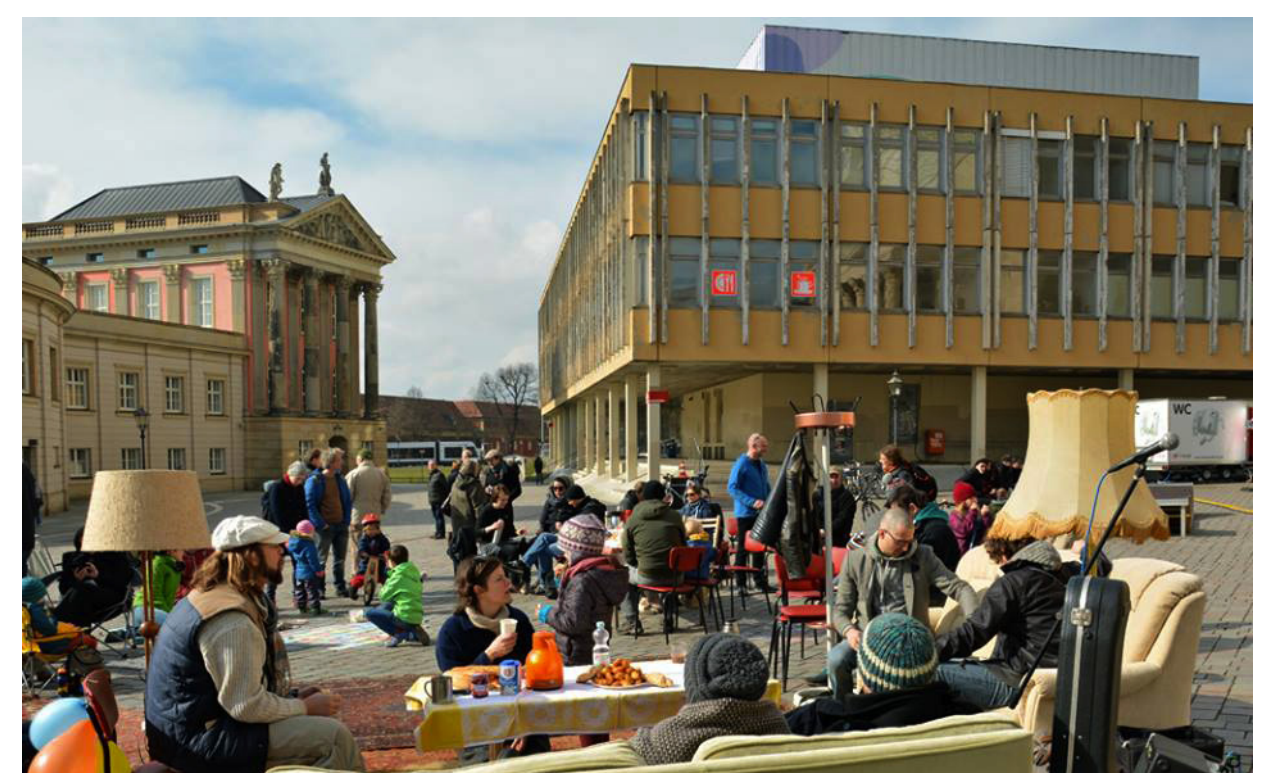


weitaus gemeinwohlorientiertere Nutzungen ermögliche als die geplanten neuen Stadtvillen. Eine Bedarfsumfrage der Initiative zeigte: Der Bedarf nach bezahlbaren und gestaltbaren Räumen für Arbeiten, Bildung, Kultur, und Wohnen im Zentrum ist enorm.

Neben den konkreten sind auch die symbolischen Dimensionen relevant, um die Mobilisierungskraft dieses Protestes zu verstehen, der sich zu einer städtischen Bewegung für eine „Stadt für Alle“ entfaltete. Die von Vielen gestellte Eigentumsfrage lässt sich deutlich ablesen an den Solidaritätsbekundungen für die Besetzer_innen der Fachhochschule: ,Das ist unsere Stadt!'skandierten Tausende in einer spontanen Demonstration. Die Infragestellung der Besitzund Entscheidungsverhältnisse hat dabei einen dezidierten Stellvertretungscharakter. Denn klar ist allen Beteiligten, dass der Erhalt der symbolkräftigen Einzelgebäude wie Fachhochschule oder Minsk weder die Frage der steigenden Mieten noch die diesem entfesselten Marktdruck zugrundeliegenden Eigentumsverhältnisse aufhalten kann. Vielmehr werden sie verstanden als letzte Bastionen, an denen noch etwas aufgehalten und verändert werden kann. Dieser Widerstand gegen den Verkauf der Stadt, in der kaum noch Perspektiven jenseits des Konsums erkennbar sind, treibt den Protest an.

Eben jene Eigentumsfrage wird aber von oben konsequent abgeblockt. Auch nach über drei Jahren Diskussion und Protest beharrt die Stadt auf dem geplanten Abriss und Verkauf städtischer Immobilien mit der mantra-artigen Rechtfertigung, dass nur so Mittel gesichert werden, um größere, neue Wohnareale am Rand der Stadt zu entwickeln. Verhandlungsspielräume für ein Moratorium kommunaler Verkäufe oder Alternativen zum Höchstpreis werden nicht eröffnet: so wurde zuletzt das Angebot des Bündnis „Stadtmitte für Alle“, die Fachhochschule über das Syndikatsmodell in Erbpacht zu kaufen und zu sanieren, trotz eines Gebots von 6 Millionen Euro als unprofessionell abgelehnt (MAZOnline 2018).

Das Beispiel der Stadtmitte zeigt damit erneut, wie eng die Privatisierungslogik mit dem Projekt der Rekonstruktion verknüpft ist: immer noch ist die Stadt Potsdam extrem zurückhaltend, wenn es darum geht, private Eigentümer_innen über die städtebaulichen - vor allem stadtbildbezogenen - Sanierungsziele hinaus in die Pflicht zu nehmen. Auflagen wie Mietpreisbindungen sind, sofern sie gestellt werden, überaus moderat.[2] Paradoxerweise sind dieselben Akteure, die seit Jahren das absolute (Verwertungs-) Primat des privaten Eigentums an Grund und Boden einfordern (siehe die Debatte um Privatisierung von Uferwegen), sehr bereit die Eigentumsfrage direkt zu stellen, wenn sie die Ziele der Rekonstruktion stören. Einmalig in der jüngeren Geschichte der Stadt wird in der Rathauskoalition tatsächlich die Forderung diskutiert, das Mercure Hotel zu enteignen und abzureißen, um die Blickachsen der barocken Stadt wiederherzustellen (Wiechers 2013).

\section{Beteiligung von oben}

Die Beteiligung der Menschen, die in der Stadt leben, ist das Paradigma nachhaltiger Stadtentwicklung. Die Stadt Potsdam versteht sich als Modellstadt für Bürgerbeteiligung und wirbt als „Beteiligungshauptstadt“ für sich. Doch was in Potsdam am deutlichsten auffällt, ist ein eklatantes Missverhältnis zwischen einem öffentlich postulierten Anspruch, mehr Beteiligung zu wagen 
und den tatsächlichen Möglichkeiten für Beteiligung sowie dem Umgang mit ihren Ergebnissen. So zeigt sich, dass Beteiligung nur zugelassen wird, wenn es den Verantwortlichen opportun erscheint - und dass diese sich an die Ergebnisse nicht gebunden fühlen, wenn sie dem eigenen Konzept nicht entsprechen. Einen Höhepunkt erreichte dieser Konflikt im Sommer 2017, als Dutzende Aktivist_innen der Initiative „Bitte stehen lassen“ die zum Abriss bestimmte Fachhochschule besetzten. Sie begründeten dies bereits im Vorfeld damit, dass ihnen keine Alternativen mehr zur Verfügung stehen, um auf ihre Anliegen aufmerksam zu machen. Prompt reagierten Stadt- und Landespolitik und Lokalmedien: „Den Besetzern sei gesagt: Werdet erwachsen und lernt, dass manchmal nicht alles so entschieden wurde, wie es Euch gefallen hätte. Man nennt das Demokratie“ (Bosschaart 2017). Ähnliches wiederholte sich im Oktober 2017 bei den Protesten gegen den öffentlichen Baustart zum Wiederaufbau der Kopie der Garnisonkirche.

Tatsächlich hat Potsdam in den letzten Jahren einige Instrumente von Beteiligung installiert. So gibt es einen Bürgerhaushalt und einen Beteiligungsrat, die Stadt fördert Engagement durch ein Büro für Beteiligung („WerkStadt für Beteiligung") und führte zu unterschiedlichen stadtpolitischen Themen Dialogverfahren durch. Aus Sicht der politisch Verantwortlichen dokumentiert sich hier der Wille, die Bürger_innen mitreden zu lassen. Ob diese dann aber politische Prozesse auch mitentscheiden können, steht auf einem ganz anderen Blatt. So erhält in jedem Bürgerhaushalt seit Jahren die Forderung ,Kein städtisches Geld für den Wiederaufbau der Garnisonkirche die große Mehrheit der Stimmen. Jetzt wurde der Bau begonnen - auf einem Grundstück, welches die Stadt der Stiftung Garnisonkirche kostenlos überlassen hat und mit der Stadt als Mitglied der Stiftung. Erst im Herbst 2017 beschloss die Stadt einen weiteren finanziellen Zuschuss an die Stiftung zum Wiederaufbau der Garnisonkirche. Inzwischen will die Stiftung auch Landesgelder akquirieren (Potsdam - Stadt für alle 2018a). Zur Entwicklung von Potsdams Mitte gab es bereits zwei Bürgerbegehren: 2014 zur Auflösung der Stiftung Garnisonkirche und 2016 gegen den Verkauf städtischer Grundstücke auf dem Gelände der Fachhochschule. Beide erhielten in wenigen Wochen mehr als die erforderlichen Stimmen, jedoch wurde ihnen mit fragwürdigen rechtlichen Mitteln Wirksamkeit versagt.

Auch das eigentlich wegweisende Projekt „WerkStadt für Beteiligung“ - in dessen Trägerverein Mitmachen e.V. ein erheblicher Teil der stadtpolitischen Initiativen mitarbeitet - wird für pro-forma-Prozesse missbraucht, bei denen die Ergebnisse bereits vorher feststehen. So kritisiert die Mieter_inneninitiative „Mieteschön Brauhausberg“, dass sie trotz langer Verhandlungen und vieler Vorschläge eigentlich nicht berücksichtigt wurden. Auch die WerkStadt für Beteiligung konstatiert: „Aber oft täuscht der Eindruck, dass noch viel zu verhandeln wäre“(Oelschläger 2017).

Bei richtig wichtigen Prozessen wird die „WerkStadt“ gar nicht einbezogen. Dafür war der „Szenarienworkshop“ zur Zukunft der Kreativwirtschaft im Frühjahr 2018 symptomatisch. Diese Workshops liefen unter dem Titel „Kunst und Kreativwirtschaft in der Potsdamer Mitte“. Für die Moderation dieser Workshops wurde ein kommerzielles Unternehmen - die UNITY AG - engagiert. Unter den zugelassenen Teilnehmer_innen war zwar die Stiftung Garnisonkirche, aber weder das Bündnis Stadtmitte für 
alle, noch die Initiative Potsdamer Mitte neu denken. Wie wenig „neutral“ (Landeshauptstadt Potsdam 2017b) die gefundene Moderation tatsächlich war, zeigte bereits der erste Workshop, als alle Ideen und Vorschläge abgelehnt worden, die über die Suche nach einem Alternativstandort für das Rechenzentrum hinausgingen. Es ging im Grunde überhaupt nicht um die öffentlich verkündete „Zukunft der Kreativwirtschaft in der Potsdamer Mitte“ (ebenda), es ging darum, das Rechenzentrum als bisherigen Standort abreißen zu können, um die Garnisonkirche wieder aufbauen zu können. Genau dies haben die Stadtverordneten dann im Juni 2018 unter Protest der am Szenarienworkshop beteiligten Kreativen dann auch beschlossen.

Alle diese Vorgänge zeigen, dass Demokratie in Potsdam als ein Entscheidungsprozess in den parlamentarischen Gremien verstanden wird. Bürger_innen können wählen. So hat es auch die Fraktion von Bündnis 90/ Die Grünen in einer Pressemitteilung zum Konflikt um die Fachhochschule festgestellt: „Eine übergroße Mehrheit der Stadtverordnetenversammlung, die durch das Mehrheitswahlrecht und weitere, kleine Gruppen [...] ein lebendiger Spiegel der Gesellschaft ist, hat dieses Ergebnis getragen.“ (Potsdam - Stadt für alle 2018b). Als „weitere, kleine Gruppen“ nehmen in Potsdam vor allem Sponsor_innen Einfluss auf dieStadtpolitik. Diese Form der Beteiligung ist in Potsdam weit wirksamer als alle Bürgerhaushalte, Bürgerbegehren oder Dialogverfahren. Ein gutes Beispiel für den Umgang mit Bürgerbeteiligung ist leider auch das sogenannte „Wohnungspolitische Konzept“. In diesem Konzept, das 2015 von der Stadt beschlossen wurde, ist auch die Aufhebung des Höchstgebotsverfahren formuliert. Doch als die städtische Gesellschaft Pro Potsdam ein Jahr später 20 Häuser zum Verkauf anbot - natürlich nach Höchstgebot - argumentierte die Stadtregierung, die städtischen Gesellschaften könnten und müssten sich nicht an solche Beschlüsse halten (Fratzke 2016). Die grundlegende Schizophrenie, dass ein nach Gesellschaftervertrag selbständiges, privatwirtschaftlich agierendes und gewinnorientiertes Unternehmen nun auch sozialpolitische Ziele erfüllen soll, bleibt eindeutig ungeklärt.

So verschieben sich in Potsdam regelmäßig die politischen Koordinaten. Längst haben die Investoren und Firmen der Stadt auch ihre eigene Wählergruppe gegründet. Selbstbeschreibend als „ohne Parteizwang, ohne Lobbyismus - dafür mit Sachverstand und Leidenschaft für die Interessen der Bürger unserer Stadt“ ist das „Bürgerbündnis Potsdam“ (Bürgerbündnis Potsdam 2018) um den Immobilieninvestor Wolfhard Kirsch eine lupenreine Klientelgruppe. Kirsch selbst verdient seit nunmehr zwei Jahrzehnten am Kauf, an der Sanierung und am Weiterverkauf von öffentlichem Eigentum Millionen. Dieser kleinen Rathausfraktion gelingt es problemlos, die regierende SPD weiter in die Richtung einer neoliberalen Stadtverwertung zu drängen. Aus dieser Perspektive verwundert es kaum, dass vor allem Lokalmedien die Bürger_inneninitiativen und Bündnisse „Stadt für alle“, „Stadtmitte für alle“ oder „Potsdamer Mitte neu denken“ regelmäßig als „links“ einordnen, obwohl diese sich selbst gar nicht so definieren. Vielmehr haben sich hier Menschen mit unterschiedlichen politischen und kulturellen Perspektiven zusammengefunden, die einfach eine andere, nachhaltige, soziale Stadtentwicklung wollen.

Das Versprechen, make Potsdam great again' bleibt politisch weitgehend unhinterfragt, muss sich kaum rechtfertigen und eignet sich offenbar dazu, 
politische Mehrheiten zu organisieren. Die strategische Annahme, dass eine Mehrheit der Wähler_innen dies als eindeutige Verschönerung und als irgendwie notwendige Wiederherstellung einer städtischen und historischen „richtigen“ Ordnung akzeptieren, hat sich bestätigt. Selbst im aktuellen Wahlkampf hat sich außer dem Kandidaten der Oppositionsfraktion „Die Andere“ keine der Bewerber_innen für den Oberbürgermeisterposten zur Sache Fachhochschule und Rekonstruktion geäußert. Allerdings erhielt dieser bei der Wahl am 23.09.2018 fast 12 \% aller Stimmen, eine Verdreifachung des Wahlergebnisses vor 8 Jahren und mehr als ein Achtungserfolg der kritischen Stadtgesellschaft.

\section{Fazit}

Vorangetrieben durch ein gut artikuliertes und begütertes politisches Netzwerk engagierter Bürger_innen hat sich das Projekt Rekonstruktion in Potsdam durchgesetzt, da es starke (Immobilien-)wirtschaftliche Interessen bedient, sich keiner planerisch begründeten Behutsamkeit unterwerfen muss und durch ein seit 1990 entwickeltes Stadterneuerungsarrangement zwischen Verwaltung, Wirtschaft und Lokalpolitik unterstützt wird. Während sich die Eliten dieser Stadt als Retter der historischen Stadt gerieren, werden die realen Gestaltungs-, Aneignungs- und Entfaltungsspielräume immer kleiner. Menschen, die eine andere Stadt wollen, werden verdrängt und zum Teil kriminalisiert.

$\mathrm{Zu}$ beachten bleibt, dass Potsdams Rekonstruktion keine rein lokale Angelegenheit ist, die sich nur lokal lösen lässt: der Einfluss nationaler Unterstützer, die Potsdams Rekonstruktion als ihrerseits wichtig finden, ist erheblich. Der vom ZDF gesponserte und von der evangelischen Kirche unterstütze Werbeclip für den Aufbau der Garnisonskirche ist exemplarisch für dieses Bestreben einer baulichen Lösung undefinierter gesellschaftspolitischer Probleme und Altlasten: der Spendenaufruf für die Errichtung dieses Gebäudes als nationaler Ort der Versöhnung kommt ohne Begründung aus, was denn genau in dieser kontaminierten Fassade versöhnt werden soll (Sagatz 2018).

Eine Lektion aus dem Beispiel Potsdam ist, dass die Analyse einer ,Stadtentwicklung von oben' auf die Wirkmächtigkeit ästhetischer Diskurse achten sollte, da sich diese als mobilisierungstauglicher, weil direkt am Alltagsgefühl anknüpfbar erweisen. Wie auch die Geschichte des Berliner Stadtschlosses zeigt, ist der Wunsch nach Versöhnung und Harmonie mit der Geschichte - oder anders gesagt die Auslöschung der Widersprüche und Untaten - ein starkes Moment. Dass sich über Ästhetik oder gar Schönheit nur schwer streiten, aber zugleich enorm viel restauratives, reaktionäres, nationales, essentialisierendes Denken transportieren lässt, macht die Potsdamer Auseinandersetzung zudem deutlich.

Natürlich geht es anders: Stadt kann bezahlbar, vielfältig und solidarisch sein. Die Umdrehung der Diskurse, also Funktion vor Form, bleibt jedoch eine Herausforderung. In derart ästhetisierten Diskursen sind neue Bilder von gemeinwohlorientierter Stadtarchitektur erforderlich. Denn, wenn Mitteschön in öffentlichen Diskussionen von der wohlproportionierten Form der preußischen Architektur spricht, die dem Harmonieempfinden des Menschen 
entspreche, werden die Kritik und die Gegenfrage, warum Demokratie feudale Fassade brauche, umgangen. Als problematisch erweist sich aber dabei, dass viele Aktive des Bündnisses „Stadtmitte für alle“ authentische Gebäude auch für schöner befinden als die monotonen Renditearchitekturen der Gegenwart. Das Defizit konkreter Bilder einer Stadt, die schön und doch für alle zugänglich ist, einer Stadt, die Brüche und Überlagerungen zeigt statt glatter einheitlicher Fassaden, wird hier deutlich. Für solche Gegenbilder erweist sich die DDR-Moderne ebenfalls als starkes, aber doch auch nur für bestimmte Gruppen ausreichendes Mobilisierungselement. Die Strategie, angebliche Verwüstungen der DDR-Zeit wiedergutzumachen bewies sich in Potsdam - auch durch die viel beschworene und reichlich verklärte Narration einer great history - zwar als die durchsetzungsstärkere; doch die den Rekonstruktionen inhärenten Widersprüche, ihre begleitenden Zerstörungen und die anhaltenden gesellschaftlichen Widerstände schreiben sich tief in die Stadtgeschichte ein.

Bislang stellt die rekonstruierte Stadtmitte den ideologischen Kitt, mit dem Widersprüche zusammengehalten und soziale Fragen ausgeklammert werden. Die Frage bleibt, wie lange es gelingt, die soziale Spaltung durch feudale Fassaden zu vertuschen? Der vollzogene Abriss der FH demonstriert, dass bislang kaum oder nur kleine Kompromisse errungen wurden (vgl. Abb. 3). Doch sind die jüngsten Auflagen sicherlich auch als ein Erfolg der Proteste zu verbuchen: Die Investoren der geplanten Stadtvillen auf historischen Grundriss müssen nun auch 15 Prozent bezahlbaren Wohnraum bereitstellen. In der aktuellen Diskussion um den Verkauf der Grundstücke auf dem Brauhausberg, verbunden mit einem Abriss des Minsk, hat sogar die Fraktion Bündnis 90/Grüne die Seiten gewechselt und verlangt eine Neuausschreibung nach Konzept. Inzwischen ist der Verkauf verschoben und eilig ein Werkstattverfahren eingeleitet worden.

Kleine Erfolge verdeutlichen den gewachsenen Widerstand gegen das lokal noch hegemoniale Projekt der Rekonstruktion. Zum ersten Mal seit über zehn Jahren haben Potsdamer_innen wieder einen konkreten Anspruch auf die Stadtmitte formuliert, der sich gegen die Pfadabhängigkeit der bisherigen Entscheidungen stemmt. Diese Gegendiskurse zeigen auf, wer in diesem großartig restaurierten Potsdam keinen Platz haben wird. Sie verteidigen hier

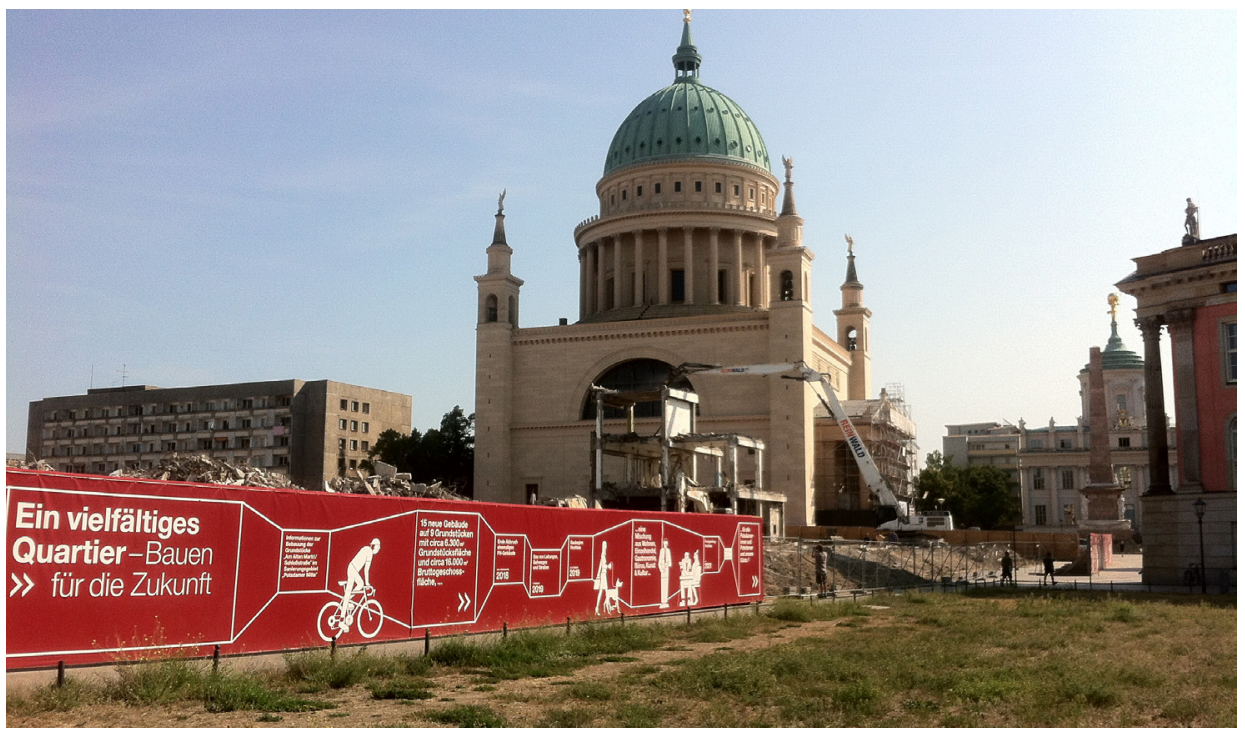

Abb. 3 Das abgerissene Gebäude der FH am Alten Markt (Quelle: Lutz 2018) 
nicht nur, sondern fordern, Neues gemeinsam zu entwickeln. Wenn ,Make Potsdam great again' das Ziel sein soll, machen sie deutlich: Großartig, vielfältig und zukunftsfähig bleibt Potsdam nur, wenn es Orte und Gelegenheiten für Mitsprache, Austausch und Begegnung schafft - und sich Räume erhält, die allen Potsdamer*innen und ihren Gästen nicht-kommerzielles Erleben, vielfältiges Miteinander und eine differenzierte Erinnerungskultur ermöglichen.

\section{Endnoten}

[1] Zum langwierigen Prozess der Form- und Funktionsfindung für den neuen Landtag bietet die Abschlussarbeit von Tomczak einen fundierten Überblick (Tomczak 2014).

[2] Laut Zeitungsmeldungen sollen in dem neuen Stadtviertel Krampnitz 20 Prozent der Wohnungen eine Mietpreisbindung haben. Bald wurde jedoch klar, dass die Stadt mit dem Großinvestor Deutsche Wohnen, der dort über 1400 Wohnungen baut, nur eine noch nicht einmal rechtlich verbindliche Zielmiete von 8,50 Euro $/ \mathrm{m}^{2}$ vereinbart hat (Oelschläger 2018).

\section{Autor_innen}

André Tomczak ist Kunsthistoriker mit Fokus auf Themen gebauter Umwelt. Er ist Mitbegründer und Sprecher der Initiativen „Potsdamer Mitte neu denken“, „Kulturlobby Potsdam“ und „Stadtmitte für alle“. Beruflich widmet er sich integrierter Stadtentwicklung, konzipiert und moderiert Beteiligungsformate und aktiviert leerstehende bzw. untergenutzte Räume. andre.tomczak@rocketmail.com

Manuel Lutz ist Stadt- und Wohnungsforscher in Berlin und Potsdam. Er forscht zum Regieren städtischer Ungleichheit und den Transformationen des Wohnens mit Fokus auf Prekarisierung und Selbstorganisation. Aktuell untersucht er neue Formen des Gemeinschaftwohnens in Deutschland und der Schweiz.

manuel.lutz@metropolitanstudies.de

Holger Zschoge ist Lehrer und Berater im Verbund des Mietshäusersyndikats. Er engagiert sich in verschiedenen städtischen Netzwerken und ist Sprecher des Bündnisses „Stadtmitte für alle“.

holger_zschoge@web.de

\section{Literatur}

AK Recht auf Stadt Potsdam (2013): Mietenstopp - Stadtumstrukturierung Verhindern - Wohnraum für Alle. http://www.rechtaufstadt-potsdam.de/ (letzter Zugriff am 17.09.2018).

Arte (2017): Metropolenreport Potsdam. https://www.arte.tv/de/videos/o75579-ooo-A/ metropolenreport-potsdam/ (letzter Zugriff am 17.09.2018).

Bosschaart, Jan (2017): Kommentar zur Besetzung der Fachhochschule Potsdam: Demokratie geht anders. In: Märkische Allgemeine Zeitung, 28.11.2107.

Bürgerbündnis Potsdam (2018): Faire Kommunalpolitik: Macht Spaß und macht Sinn. https://www.buergerbuendnis-potsdam.de/buergerbuendnis/ueber-uns.html (letzter Zugriff am 17.09.2018).

Calvez, Sandra (2018): Studenten protestieren für mehr Wohnraum: Bezahlbar wohnen in Potsdam? In: Potsdamer Neueste Nachrichten, 6.6.2018.

Förderverein Wiedergewinnung der Potsdamer Mitte e.V. (2018): Mitteschön! - Initiative „Bürger Für Die Mitte“. https://www.mitteschoen.de/ (letzter Zugriff am 17.9.2018). 
Fratzke, Christine (2016): Nein für Tuchmacher 8. In: Potsdamer Neueste Nachrichten, 4.11.2016.

FreiLand (2018): FreiLand Potsdam Kulturzentrum. In: https://www.freiland-potsdam.de/ (letzter Zugriff am 17.9.2018).

Gellner, Torsten (2018): Wohnungsmarkt: Potsdam Bleibt Das Teuerste Pflaster in Ostdeutschland. In: Märkische Allgemeine Zeitung, 19.2.2018.

Hallo Potsdam (2017): Hallo Potsdam: Lebensqualität und Stadtentwicklung aus kritischer Perspektive. https://stadtfueralle.de/wp-content/uploads/hallopotsdam_2017.pdf (letzter Zugriff am 17.9.2018).

Helbig, Marcel / Jähnen, Stefanie (2018): Wie brüchig ist die soziale Architektur unserer Städte? Trends und Analysen der Segregation in 74 deutschen Städten. "Working Paper P 2018-001, Wissenschaftszentrum Berlin für Sozialforschung, Berlin.

Kil, Wolfgang (2017): Adieu Brauhausberg! Potsdams neues Freizeitbad erweist der Stadt einen schlechten Dienst. https://www.baunetz.de/meldungen/Meldungen-Potsdams_ neues_Freizeitbad_erweist_der_Stadt_einen_schlechten_Dienst_5099445.html (letzter Zugriff am 17.9.2018).

Kramer, Henri (2017): Demo gegen hohe Mieten: mit markigen Worten gegen Verdrängung. In: Potsdamer Neueste Nachrichten, 16.9.2018.

Landeshauptstadt Potsdam (1990): Beschluss der Stadtverordnetenvollversammlung zur behutsamen Wiederannäherung an den charakteristischen, historisch gewachsenen Stadtgrund- und Aufriß.

Landeshauptstadt Potsdam (2017a): Wohnungsmarkt Potsdam Soziale Wohnraumversorgung in der Landeshauptstadt Potsdam 2013 bis 2016.

Landeshauptstadt Potsdam (2017b): Pressemitteilung: Szenario-Workshop: Strategieentwicklung für die Kunst- und Kreativwirtschaft in der Potsdamer Mitte.

Maak, Niklas / Seidel, Claudius (2017): Make Potsdam Schön Again. In: Frankfurter Allgemeine Zeitung, 10.4.2017.

MAZOnline (2018): Potsdam lehnt Kaufangebot für FH ab. In: Märkische Allgemeine Zeitung, 2.5.2018.

Oelschläger, Volker (2014): Vom Archiv bis zum Spartacus. In: Märkische Allgemeine Zeitung, 10.01.2014.

Oelschläger, Volker (2017): Altbausanierung in Potsdam - Zwischenbilanz zur Mieterbeteiligung. In: Märkische Allgemeine Zeitung, 14.07.2017.

Oelschläger, Volker (2018): Krampnitz Ohne Mietobergrenze. In: Märkische Allgemeine Zeitung, 15.7.2018.

Pietryga, Sophia (2018): Gestern - Rechenzentrum Potsdam. https://rz-potsdam.de/cms/ gestern/ (letzter Zugriff am 17.9.2018).

Potsdam - Stadt für alle (2018a): Garnisonkirche - ein Goldenes Kalb für Potsdam. http:// www.potsdam-stadtfueralle.de/2018/o9/12/garnisonkirche-ein-goldenes-kalb-fuerpotsdam/ (letzter Zugriff am 17.9.2018).

Potsdam - Stadt für alle (2018b): Zitate zur Debatte um die Fachhochschule Potsdam. http://www.potsdam-stadtfueralle.de/2018/o3/10/zitate-zur-debatte-um-die-fachhochschule-potsdam/ (letzter Zugriff am 17.9.2018).

Potsdamer Mitte neu denken (2016): Haus der Gegenwart. https://www.potsdamermitteneudenken.de/haus-der-gegenwart/ (letzter Zugriff am 17.9.2018).

rbb (2017): Wem gehört Potsdams Mitte? https://mediathek.rbb-online.de/tv/ Die-rbb-Reporter/Wem-gehört-Potsdams-Mitte/rbb-Fernsehen/Video?bcastId=16269336\&documentId=48161760 (letzter Zugriff am 17.9.2018).

Sagatz, Kurt (2018): Spot für Garnisonkirche bringt ZDF Ärger ein. In: Der Tagesspiegel, 21.02.2018.

Tillack, Hans-Martin (2011): Immobiliendeals in Brandenburg: Wie Platzeck Potsdam verkaufte. In: Der Stern, 27.9.2011.

Tomczak, André (2014): Von der kritischen Rekonstruktion zur ,Mitteschön!‘ Die Fassadenrekonstruktion des Stadtschlosses im Diskurs der Potsdamer Mitte. Technische Universität Berlin. https://depositonce.tu-berlin.de/handle/11303/6276 (letzter Zugriff am 17.9.2018).

Wiechers, Katharina (2013): Stadt fällt mit Plänen für Mercure-Abriss durch. In: Potsdamer Neueste Nachrichten, 30.10.2013.

Zschiek, Marco (2018): Potsdam unter Deutschlands zehn teuersten Städten. In: Potsdamer Neueste Nachrichten, 16.7.2018. 